\title{
Pump-Probe Experiments at X-ray Free Electron Laser Sources
}

Femtosecond Dynamics of the Collinear-to-Spiral Antiferromagnetic Phase Transition in $\mathrm{CuO}$

Authors: S. L. Johnson, R. A. de Souza, U. Staub, P. Beaud, E. Mo"hr-Vorobeva, G. Ingold, A. Caviezel, V. Scagnoli, W. F. Schlotter, J. J. Turner, O. Krupin, W.-S. Lee,Y.-D. Chuang, L. Patthey, R. G. Moore, D. Lu, M. Yi, P. S. Kirchmann, M. Trigo, P. Denes, D. Doering, Z. Hussain, Z.-X. Shen, D. Prabhakaran, and A. T. Boothroyd Physical Review Letters 108, 037203 (2012)

Driving magnetic order in a manganite by ultrafast lattice excitation Authors: M. Forst, R. I. Tobey, S. Wall, H. Bromberger, V. Khanna, A. L. Cavalieri, Y.-D. Chuang,W. S. Lee, R. Moore, W. F. Schlotter, J. J. Turner, O. Krupin, M. Trigo, H. Zheng, J. F. Mitchell, S. S. Dhesi, J. P. Hill, and A. Cavalleri Physical Review B 84, 241104 (R) (2011)

\section{Recommendation and commentary by Sunil Sinha, UCSD}

X-ray Free Electron Lasers (XFEL's) have now established themselves as the next generation of super-brilliant X-ray sources, with peak brilliances as high as ten orders of magnitude greater than the brightest synchrotron storage-ring based sources, delivering photon beams with a high degree of transverse coherence in pulses as short as $10-200$ femtoseconds. The Linac Coherent Light Source (LCLS) at Stanford was the first hard X-ray XFEL to operate successfully and currently has a flourishing user program. Similar sources are about to come online at the DESY laboratory in Hamburg(which has operated an extreme UV prototype FEL called FLASH for several years) and at the Spring-8 synchrotron facility in Japan. Korea and China are also planning to build XFEL's. These sources, which are LINACbased, work on the so-called SASE (Self-Amplified Spontaneous Emission) process, which we do not have space to discuss in this article. However numerous references are available [1].

It is natural that the unique properties of the XFEL should lend themselves to studies which are not easily carried out at conventional synchrotron sources, where the photon pulses are picoseconds in duration, and the coherence fraction is much lower. The types of publications coming from LCLS involve femtosecond nanocrystallography [2], speckle reconstruction imaging [3], fast highly non-linear effects in atoms (e.g. the creation of "hollow atoms" via the rapid ionization of the inner shells [4]) and pump-probe type studies of non-equilibrium phenomena in 
solids, of which the papers discussed in this article are good examples. As one can see from the author lists, the teams of people who need to get together for one experiment at the XFEL are getting quite large, although nowhere near the size of teams for high-energy physics experiments!

The availability of lasers capable of producing intense short ( $\sim 10 \mathrm{fs}$ to $100 \mathrm{fs}$ ) pulses of light from the mid infra red to the visible wavelength range made it possible to hit a sample with a laser pulse (the pump) and interrogate after a fixed delay with another pulse (the probe). This kind of technique has, for instance, led to the field of femtochemistry. With the advent of short X-ray pulses from XFEL's, it is now possible to pump with a laser and probe with an X-ray pulse, thereby gaining the ability to study the effects of the perturbing pulse on short length scales, i.e. the effect on crystalline or magnetic order. This synergy between two very different laser technologies, one in the infrared or visible, and the other in the X-ray regime has turned out to be very scientifically profitable at the new sources. The challenges associated with phasing the delay of the X-ray probe pulse to the laser pump pulse should not be underestimated, but it appears that the technology has been mastered, so that system responses can be measured on femtosecond timescales after the pump.

The need for ultra high-speed switching of magnetization of bits in magnetic storage devices has led to several studies of switching using intense short magnetic pulses, etc. The most promising method appears to be to use ultrafast and intense laser pulses, where demagnetization of ferromagnets can be achieved on femtosecond timescales. This has led to the so-called field of femtomagnetism.

The papers discussed here are not concerned directly with the effect of laser pulses on ferromagnetic order, but on antiferromagnetic order and orbital order on femtosecond timescales. These require probes of much shorter wavelengths than optical probe pulses. The probe is resonant X-ray diffraction from the magnetic ordering, which occurs away from the conventional Bragg peaks of the crystal structure.

In the paper by Johnson et al., the laser excitation is applied close to a known phase transition to drive a $\mathrm{CuO}$ crystal from a low-temperature commensurate and collinear antiferromagnetic (AF) phase to an incommensurate (IC) spiral spin structure (which contains a small ferroelectric moment) which in equilibrium exists at a slightly higher temperature. The laser excitation promotes a non-thermal spin disorder, which drives the material into the IC phase. In actual fact, owing to the first order nature of the transition, there is coexistence of the two phases at the 
temperature of the experiment $(207 \mathrm{~K})$, but with only a small fraction of the IC phase. This becomes the major fraction after the laser excitation pulse. This pulse causes both peaks to suffer an initial decrease on a time scale of less than $300 \mathrm{fs}$, the mechanism for which is unknown at the present time. There is a $\sim 2$ ps delay in the time at which the ratio of the intensity of the driven IC phase peak relative to the commensurate AF peak starts to increase relative to the exciting laser pulse. This time turns out to decrease with increasing fluence of the exciting laser pulse, saturating at $\sim 400$ psecs at high fluences. The authors give a rough argument that this corresponds to a quarter period of a spin wave excitation whose wavevector is the "coordinate" that takes the free energy of the system from one phase to the other, as in discussions of the time scales for structural transitions.

In the paper by Forst et al., a different approach was taken. The sample was a singlelayer manganite $\mathrm{La}_{0.5} \mathrm{Sr}_{1.5} \mathrm{MnO}_{4}$, in which both magnetic spin and orbital order exist at low temperatures. The exciting pulses were femtosecond pulses of mid-infrared laser light tuned to a center wavelength of $13.5 \mu \mathrm{m}$ but with a wavelength spread broad enough to excite the Mn-O stretching vibration at $16 \mu \mathrm{m}$. The magnetic spin order and orbital order were resonantly probed with femtosecond x-ray pulses tuned to the $\mathrm{Mn} \mathrm{L}_{3}$-edge. By comparing their results to excitations with light pulses of 800$\mathrm{nm}$ wavelength, they showed that the magnetic order with the mid-infrared exciting pulse is much slower, with an $8 \%$ reduction with a time constant of $12.2 \mathrm{ps}$ as compared to $250 \mathrm{fs}$ for the optical exciting pulses. They also showed that the orbital order is degraded with a faster time constant of $6.3 \mathrm{ps}$, although to a lesser extent (3 $\%)$. This hierarchy of time scales is explained in terms of the mid-infrared pulse coupling to the collective Jahn-Teller distortion modes, thus destroying first the orbital and then the spin order, while the near-infrared pulses excite charge transfer across semicovalent bonds, destroying the spin order on femtosecond time scales.

\section{References}

[1] R. Bonifacio, L. De Salvo, P. Pierini, N. Piovella, and C.

Pellegrini, "Spectrum, temporal structure, and fluctuations in a high gain free electron laser starting from noise" Phys. Rev. Lett. 73 (1994), p. 70.

[2] A. Aquila et al., "Time-resolved Protein Nanocrystallography Using an X-ray Free-electron Laser" Optics Express 20, 2706-2716 (2012)

[3] J. Miao, P. Charalambous, J. Kirz, and D. Sayre, "Extending the methodology of $\mathrm{X}$-ray crystallography to allow imaging of micrometre-sized non-crystalline specimens", Nature 400, 342 (1999)

[4] G. Doumy et al., "Nonlinear Atomic Response to Intense Ultrashort X Rays" Phys. Rev. Lett. 106, 083002 (2011) 